\title{
Magnetic structure of Fe-Fe oxide nanoparticles made by electrodeposition
}

\author{
Santosh Kumar ${ }^{1}$, Samar Layek², Brajesh Pandey ${ }^{2}$ and H.C. Verma ${ }^{2, *}$ \\ ${ }^{1}$ Department of Physics, B S College, Danapur, Bihar, INDIA. \\ ${ }^{2}$ Department of Physics, Indian Institute of Technology, Kanpur 208016, INDIA. \\ "Corresponding Author: H. C. Verma, e-mail: hcverma@iitk.ac.in
}

\begin{abstract}
Iron/iron-oxide nano particles are synthesized using electrodeposition technique. The particles are well crystalline bcc iron with disordered oxides of iron on the surface. The particles show very small coercivity displaying superparamagnetic behavior of the iron core in magnetization measurement using Vibrating Sample Magnetometer. But the same particles show no trace of supermagnetism or fluctuation of magnetic moments in Mössbauer measurements, showing the role of time scales of measuring equipment in fine particle magnetism.
\end{abstract}

Keywords: Iron/iron oxide composite, Magnetic nanoparticles, VSM, Mössbauer spectroscopy.

\section{Introduction}

Magnetic nanoparticles have been a major research area during recent times. This is due to a variety of applications they offer such as information storage (Kozicki et al, 2003), magneto optical devices (Kamat, 2002; Klein et al., 2005), magnetic fluids (Barclay, 1982), targeted drug delivery (Gangopadhyay et al., 2005; Dobson, 2006), sensing devices (Roumenin, 1994) and so on. Novel processing procedures have been developed for the synthesis of such materials and it has been established that process parameters sensitively control the structural evolution and result in newer properties (Dumitrache et al., 2005). At nanosize, the defects in the structure, the shape of the particle etc. are much more effective in altering the properties as compared to their bulk counterparts. Metal - metal oxide junctions in nanosystems could be an interesting object. For example, cobalt nanoparticles with cobalt oxide at the surface results in a magnetic system with unidirectional anisotropy instead of uniaxial anisotropy (Meiklejohn and Bean, 1957). This is supposed to come from the exchange bias interaction between ferromagnetic cobalt and antiferromagnetic cobalt oxide. Many more studies are reported on such ferromagnetic / antiferromagnetic systems including $\mathrm{Fe} / \mathrm{FeO}$ core shell structure (Gangopadhayay et al., 1991; Bianco et al., 1998; Presting and Konig, 2003; Hilger et al., 2004; Shah and Basu, 2008). Also metals are electrically conducting whereas oxides are mostly insulators. Metals with their surface oxidized are natural metalinsulator junction which may find applications in magnetoelectronic devices (Bianco et al, 1998; Presting and Konig, 2003), optical filters (Shah and Basu, 2008), biomedical materials (Hilger et al, 2004) etc.

Gangopadhyay and co-authors (Gangopadhayay et al, 1991) reported formation of iron nanoparticles with oxide coating using evaporation and condensation of metal in inert atmosphere. The coercivity was found to be much more in the oxide coated particles as compared to the case with pure iron. Fe / FeO particles were synthesized using laser pyrolysis by Dumitrache et al. (2005) with mean core size of $14 \mathrm{~nm}$ and shell thickness $4 \mathrm{~nm}$ and were shown to agglomerate under their mutual magnetic interaction. The nanoparticles in these studies showed good crystallinity.

In all these studies, the surface was shown to consist mainly of $\alpha-\mathrm{Fe}_{2} \mathrm{O}_{3}, \gamma-\mathrm{Fe}_{2} \mathrm{O}_{3}$ and $/$ or $\mathrm{Fe}_{3} \mathrm{O}_{4}$. These phases were detected using SAED pattern in TEM (Dumitrache et al, 2005) and Mössbauer spectroscopy (Gangopadhayay et al, 1991). Taking the nanoparticles synthesis from laboratory scale to mass production is quite important in view of their application potential. We have used a totally different approach to synthesize iron-iron oxide nanoparticles and have used a wet method based on electrolysis. Compared to previous processes discussed above, it's very cheap, simple to assemble, much less time taking and easily scalable for large quantity production. The particles so prepared show quite interesting properties, different from what has been observed for particles made from metal vapour state. Most interestingly, our particles show superparamagnetic behavior in magnetization measurements and ferromagnetic behavior in Mössbauer spectroscopic measurements. 


\section{Materials and Methods}

Pure iron powder was synthesized by two-electrode electrodeposition in an electrolytic bath. A rectangular gold plate $(4 \mathrm{~cm} \times 2.5$ $\mathrm{cm})$ was used as anode and a pure aluminium substrate of the same size as cathode. $74.60 \mathrm{gm}$ of $\mathrm{FeSO}_{4} .7 \mathrm{H}_{2} \mathrm{O}(\mathrm{MERCK}$, purity $>99.9 \%$ ) was dissolved in $250 \mathrm{ml}$. of deionized water at room temperature to give the electrolytic bath. Electrodeposition was done at constant voltage of $5 \mathrm{~V}$ for 5 minutes after which the aluminium substrate was removed from the bath and allowed to dry. The material deposited on the substrate was gently scrapped and was allowed to oxidize naturally in open atmosphere. This powder has been used in all characterization experiments. Using weight measurements of the substrate before and after the deposition, it was found that about $23 \%$ of the initial iron could be recovered as $\mathrm{Fe}$ nanoparticles. The sample so prepared was subjected to X-ray diffraction on a Seifert Iso-Debyeflex 2002 diffractometer using $\mathrm{Cr}-\mathrm{K}_{\alpha}$ radiation (wavelength =2.2898 $\AA$ ) to see the crystallographic structure formed. Small amount of sample was gold deposited and investigated using scanning electron microscopy (ZEISS, SUPRA 40VP) in order to see particle morphology and average crystallite size.

Mössbauer spectra of the sample were recorded using a conventional constant acceleration Mössbauer spectrometer. ${ }^{57} \mathrm{Co}$ in a rhodium matrix was used as the radioactive source. Low temperature spectra were recorded using a closed cycle cryostat. The data were analyzed using a least squares method assuming Lorentzian line shapes. Room temperature magnetization measurement was carried out using a vibrating sample magnetometer (ADE Magnetics, USA) upto an applied field of 1.75T.

\section{Results and Discussion}

\subsection{X-ray diffraction}

The XRD pattern shows a well crystalline iron structure. It does not indicate any oxide peaks. This is because the oxides formed at the surface are likely to be disordered. Spectrum of bcc iron (Aldrich, 99.99\% pure) powder of bulk size is also shown for a reference. The line width of the XRD peak of electrodeposited particle is much larger than that of the bulk powder as can be seen visually. Calculation based on Scherrer formula, after correcting for instrumental broadening obtained from the bulk size XRD pattern, gives the average crystallite size to be $\sim 24 \mathrm{~nm}$.

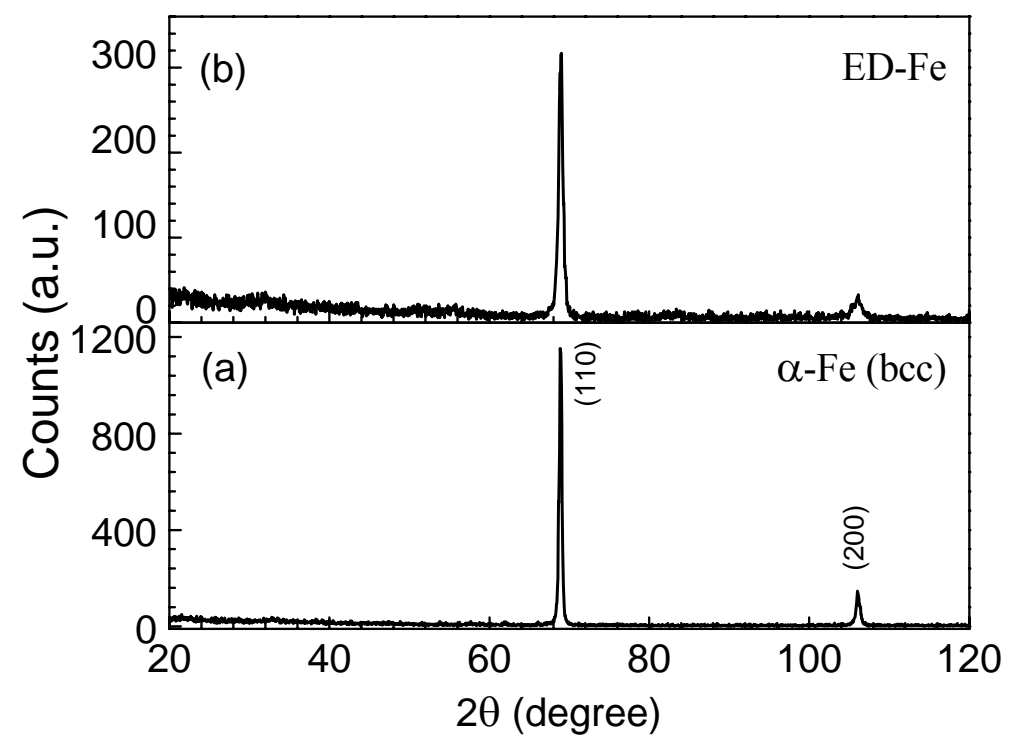

Figure 1 Room temperature X-ray diffraction pattern of (a) bulk $\alpha$-Fe particles (bcc phase) (b) electrodeposited iron/iron oxide nanoparticles and with $\mathrm{Cr}-\mathrm{K} \alpha$ radiation.

\subsection{Scanning Electron Microscopy (SEM)}

Figure 2 shows the SEM micrograph. As no dispersion of particles was done prior to making SEM specimen, these are agglomerated to each other. However, the particle boundaries in the polished sample are quite clear. The particles seem to have nonspherical irregular shape and the size range is roughly from 20 to $60 \mathrm{~nm}$. This is consistent with XRD result and shows that particles are formed mostly in single crystallite form. 


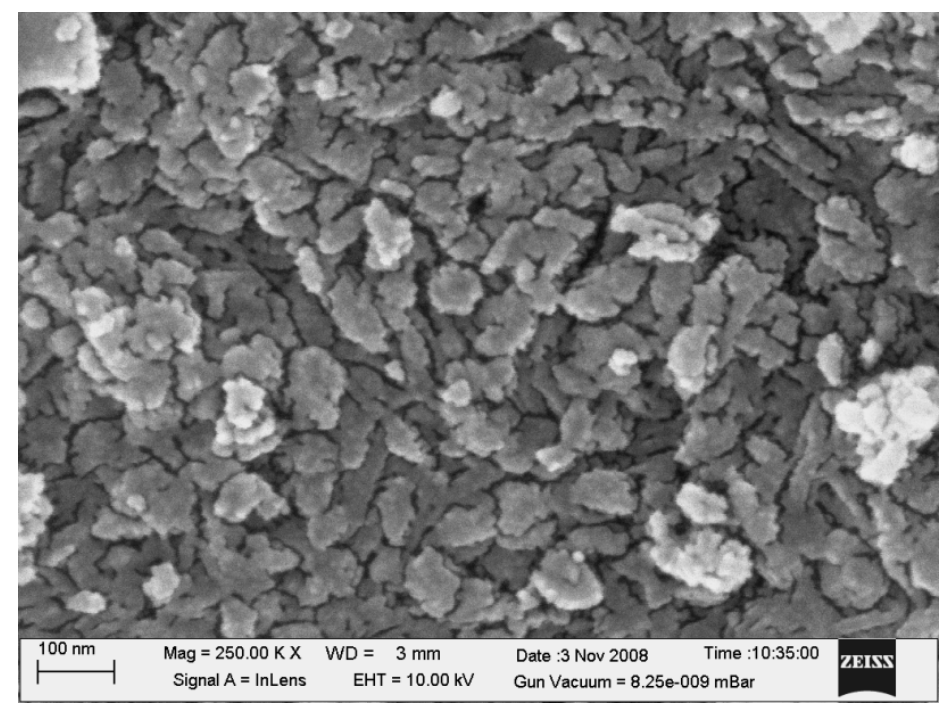

Figure 2 SEM image of electrodeposited iron/iron oxide nanoparticles.

\subsection{Mössbauer Spectroscopic Studies}

Mössbauer spectroscopy gives the information about immediate neighborhood of ${ }^{57} \mathrm{Fe}$ probe nuclei and is much more sensitive than X - ray diffraction technique. Another great advantage with Mössbauer spectroscopy is that its time scale for measurement is $10^{-8} \mathrm{~s}$ which compares well with the superparamagnetic relaxation time scale in nanoparticles (Tuček et al, 2006). This helps in understanding the magnetic dynamics much better. The Mössbauer spectrum of the sample recorded at room temperature $295 \mathrm{~K}$ is shown in Figure 3 (a). One can see that the spectrum is a superposition of a sharp sextet and a doublet.

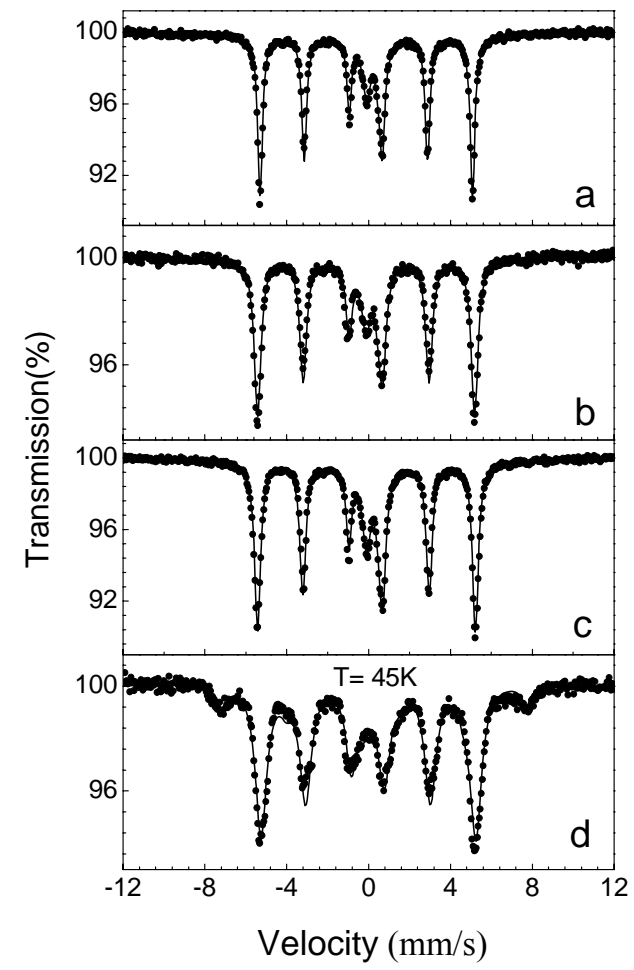

Figure 3 Room temperature Mössbauer spectra of the electrodeposited iron/iron oxide nanoparticles. (a) Fresh sample (b) 5 months old sample and (c) 11 months old sample and (d) Spectrum at $45 \mathrm{~K}$.

The Mössbauer parameters given in Table 1 reveal that the sample contains well crystalline bcc phase of iron and ferromagnetic domains are well grown. The parameters of the sextet, namely hyperfine magnetic field of $33.1 \mathrm{~T}$, Isomer shift and Quadrupole Splitting almost zero, match exactly with those of a bulk crystalline bcc iron sample. The nanosize effect like superparamagnetism 
or collective magnetic excitation are almost absent in the Mössbauer spectrum. For Mössbauer spectroscopy, the particles behave as bulk particles. The line width of sextet components is $0.35 \mathrm{~mm} / \mathrm{s}$ which is bit larger than the width obtained with our setup in pure phase components $(0.28 \mathrm{~mm} / \mathrm{s})$. The extra broadening may come due to defect productions which are known to exist in large numbers in electrodeposited materials (Roy et al., 2002; Roy et al, 2004). Together with the sextet we have a doublet component having about $23 \%$ of the total absorption area. The doublet signifies that a portion of Fe atoms are not magnetically coupled, i.e., the matrix consists of other elements around Fe atoms. This two-line pattern is indicative of some oxide formation at the surface. The Isomer Shift of $0.34 \mathrm{~mm} / \mathrm{s}$ shows that iron is in $\mathrm{Fe}^{3+}$ state in this nonmagnetic part. The line width of this component is rather large, $0.50 \mathrm{~mm} / \mathrm{s}$, showing that the surface oxidized part does not correspond to a single phase. The usual corrosion products of iron in wet atmosphere contain $\alpha-\mathrm{FeOOH}, \mathrm{Fe}_{3} \mathrm{O}_{4}, \gamma-\mathrm{Fe}_{2} \mathrm{O}_{3}$ and finally $\alpha-\mathrm{Fe}_{2} \mathrm{O}_{3}$. In their bulk form they all give six-line pattern in Mössbauer spectroscopy. In the present case, one only has a doublet corresponding to the oxidized $\mathrm{Fe}^{3+}$ part showing that the oxide layer has much shorter range of ordering.

Table 1. Mössbauer parameters of electrodeposited iron-ironoxide nanoparticles at different time at $300 \mathrm{~K}$ and for fresh sample at $45 \mathrm{~K}$.

\begin{tabular}{|c|c|c|c|c|}
\hline Sample & $\begin{array}{c}\text { IS (mm/s) } \\
\pm 0.02 \mathrm{~mm} / \mathrm{s}\end{array}$ & $\begin{array}{c}\text { QS }(\mathrm{mm} / \mathrm{s}) \\
\pm 0.02 \mathrm{~mm} / \mathrm{s}\end{array}$ & $\begin{array}{c}\mathrm{B}_{\mathrm{hf}}(\mathrm{T}) \\
\pm 0.1 \mathrm{~T}\end{array}$ & $\begin{array}{c}\text { Area (\%) } \\
\pm 2 \%\end{array}$ \\
\hline Fresh (295 K) & 0.34 & 0.70 & - & 23 \\
& -0.02 & 0.01 & 33.1 & 77 \\
\hline 5 months old (295 K) & 0.31 & 0.68 & - & 19 \\
& -0.03 & 0.00 & 32.9 & 81 \\
\hline 11 months old (295 K) & 0.33 & 0.68 & - & 21 \\
& -0.03 & 0.01 & 32.7 & 79 \\
\hline Spectrum at 45 K & 0.29 & 0.90 & - & 9 \\
& 0.07 & 0.01 & 32.6 & 79 \\
& 0.41 & -0.02 & 46.7 & 12 \\
\hline
\end{tabular}

From the Mössbauer pattern one can envisage the sample to have particles with well crystalline iron in bcc phase composited with oxides of iron without long range ordering. The oxide formation can be from the atmosphere after the electrode is exposed to air or during the deposition and crystallization stage itself taking oxygen from the solution. In the previous case the oxide will make a shell around the iron particle. If we assume the same recoil-free fraction and the same atom density of Fe in the two components, we can estimate the ratio of shell thickness to core thickness. A simple calculation gives this ratio to be about 0.1 . However, this is an underestimation of shell thickness because the iron atom density in the oxide will be much smaller than that in the iron core. All these calculations show that the surface to volume ratio is quite large indicating that the particles are of fine size consistent with the SEM findings $(20-60 \mathrm{~nm})$. Another possibility is that the oxidation has gone deeper into the particle. The magnetization measurement however supports the former presumption as described later.

The environmental stability is an important issue in using iron nanoparticles. The prepared sample was naturally oxidized in open atmosphere and the surface seemed to be well passivated. The first measurements of XRD and Mössbauer spectroscopy discussed above were performed within a few days of formation of the particles. Room temperature Mössbauer spectrum and XRD of the same sample were twice repeated at intervals of about six months each. The Mössbauer spectra are shown in Figures 3 (b) and (c) and the parameters are included in Table 1. The spectra give almost the same sextet - doublet pattern with similar area ratio. The XRD patterns are also giving exactly the similar pattern. Usually iron is very reactive and one would expect greater corrosion with time but this electrodeposited iron is quite stable for at least one year and no further oxidation had taken place in this period.

In order to see the doublet component further we recorded Mössbauer spectrum of Fe/FeO sample at $45 \mathrm{~K}$ (Figure $3 \mathrm{~d}$ ). A part of the doublet component splits into magnetic six-line pattern of hyperfine field $46.7 \mathrm{~T}$. The absorption area corresponding to the iron remains the same. The area compositions are given in Table 1. From the parameters, formation of $\mathrm{Fe}_{3} \mathrm{O}_{4}$ in any significant amount is ruled. The sextet peaks corresponding to the oxide phase are not sharp showing that these are formed in different size range, not well crystalline and may be with lots of defects.

\subsection{Magnetic properties}

Figure 4 shows the M-H curve recorded using VSM with a maximum field of $1.75 \mathrm{~T}$. It is seen that the saturation magnetization $\mathrm{M}_{\mathrm{s}}$ is $155 \mathrm{emu} / \mathrm{g}$ which is about $70 \%$ of the standard value $220 \mathrm{emu} / \mathrm{g}$ for pure iron phase. It is interesting to note that the absorption area under the sextet in the Mössbauer spectrum is also of the same order (77\%). 


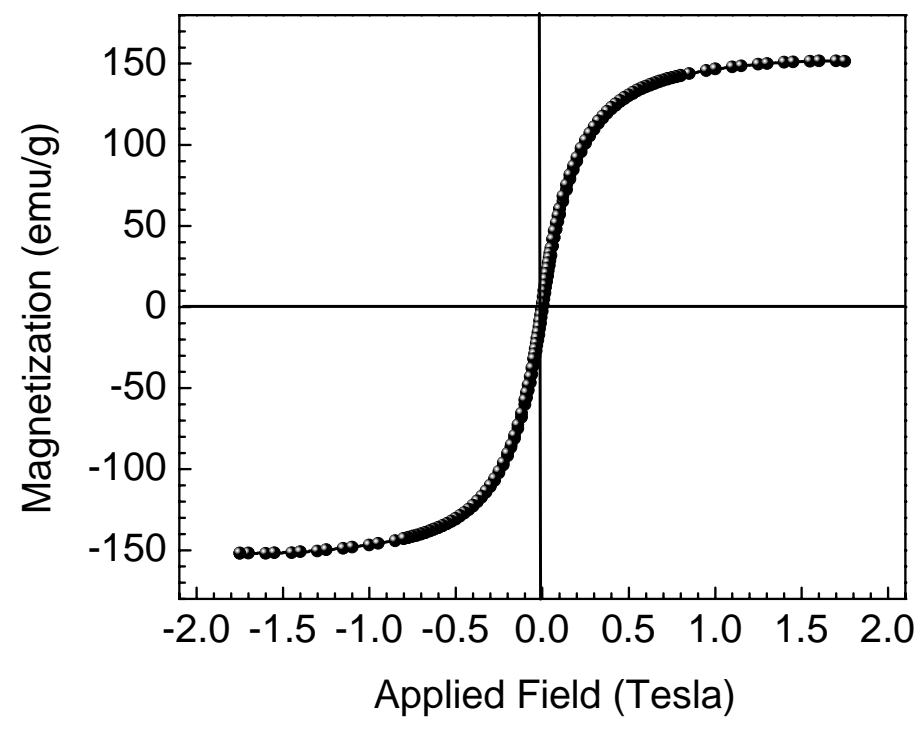

Figure 4 Isothermal M-H curve of the electrodeposited iron/iron oxide nanoparticles at room temperature upto an applied field $1.75 \mathrm{~T}$.

So the magnetization seems to come only from the well crystalline bcc iron phase and the oxide part is not contributing anything to it. The most interesting observation is that the hysteresis loop has not opened up. Coercive field is almost zero. This observation is suggestive of the fact that in the time scale of magnetization measurement, the sample is behaving like a superparamagnetic material. From the XRD and SEM we inferred that they are single crystallite particles. And almost zero coercive fields tell us that iron particles are mostly single domain particles. We conclude that the size range of Fe particles $(\sim 24 \mathrm{~nm})$, behaves as superparamagnetic in the magnetization measurement but as bulk in Mössbauer measurement. This comes about because the measurement time scales for the two experiments are very different, being few seconds for VSM and around $10^{-8} \mathrm{~s}$ for Mössbauer measurement. The superparamagnetic fluctuation time, in which the magnetic moment reverses its orientation due to thermal energy, is given as $\tau=\tau_{0} * \exp (-\mu \mathrm{V} / \mathrm{KT})$ and depends sensitively on the particle size (volume $\mathrm{V}$ ). For our particles, this time seems to be much larger than measuring time of Mössbauer spectroscopy but much smaller than that of magnetization measurement.

It is interesting to note that in one of the studies of iron-iron oxide core shell structure by Crisan et al., (2008) the particles are of 4 to $6 \mathrm{~nm}$ size and the magnetization curve at room temperature shows a strong ferromagnetic character with sharp approach to saturation of the magnetization and marked value of coercivity. The particle size of our synthesized material is much larger than this but still it shows superparamagnetic behavior in magnetization measurement at room temperature. This difference behavior may be attributed to the very different process of synthesis i.e., electrodeposition.

\section{Conclusions}

1. A composite structure having a well crystalline bec iron coexisting with disordered iron oxide is formed by the easily accessible electrolysis process. X-ray diffraction and Mössbauer spectroscopy show a well crystalline bcc iron phase with crystallite size of the order of $24 \mathrm{~nm}$. The oxide formation takes place in much smaller size and with lack of crystallinity, corresponding to a broad doublet in Mössbauer spectrum which partially splits at low temperature. No indication of oxides is given by XRD showing that the material in the oxide layer is disordered.

2. The magnetization measurement gives a saturation magnetization of about $70 \%$ of the standard value for bulk iron. It compares well with the law of dilution assuming that the oxide parts do not contribute to magnetization and the bcc iron gives full magnetization corresponding to iron.

3. The most interesting observation is that the samples gives a sharp six line pattern with undiminished $\mathrm{B}_{\mathrm{hf}}$ of $33 \mathrm{~T}$ corresponding to bulk iron phase, but the same sample gives almost zero coercive field in $\mathrm{M}-\mathrm{H}$ measurement as if the sample is superparamagnetic. The particle size, as shown by XRD and SEM pictures, is in the intermediate range (20$60 \mathrm{~nm}$ ), so that, at the time scale of Mössbauer spectroscopy, the magnetic moments behave as steady but at the much longer time scale of magnetization measurement they fluctuate and behave as superparamagnetic. Still the behaviour is unusual, in the sense that the saturation magnetization does not seem to be reduced and follows the law of dilution. The measurements are quantitatively repeatable even after one year of formation of the sample, though no special protective environment was provided. 
Nanoparticles are being increasingly used in different domains of human use and issues like their environmental impacts are seriously looked into (Nowack and Bucheli, 2007). Stability of nanoparticles and their reactivity in environment are therefore important. The finding of behaviour of iron/iron oxide nanoparticles prepared through electrodeposition is therefore interesting.

\section{Acknowledgement}

One of the authors, Santosh Kumar, gratefully acknowledges financial support from University Grants Commission under Minor Research Project - PSB - 009 / $05-06$ and the central nuclear laboratory facility of IIT, Kanpur. Special thanks are given to Pratyush Vaibhav, for effective cooperation. We acknowledge help received from Dr Dinesh Deva of Chemical Engineering Dept, IIT Kanpur in recording SEM micrographs.

\section{References}

Barclay J. A., 1982. Use of a ferrofluid as the heat-exchange fluid in a magnetic refrigerator. Journal of Applied Physics, Vol. 53, pp. 2887-2894.

Bianco L.D., Hernando A., Multigner M., Prados C., Sànchez-López J. C., Fernández A., Conde C. F., and Conde A., 1998. Evidence of spin disorder at the surface-core interface of oxygen passivated Fe nanoparticles. Journal of Applied Physics, Vol. 84, pp. 2189-2192.

Crisan O., Haeften K.V., Ellis A.M. and Binns C., 2008. Structure and magnetic properties of Fe/Fe oxide clusters. Journal of Nanoparticle Research, Vol. 10, pp. 193-199.

Dobson J., 2006. Magnetic nanoparticles for drug delivery. Drug Development Research, Vol. 67, pp. 55-60.

Dumitrache F., Morjan I., Alexandrescu R., Ciupina V., Prodan G., Voicu I., Fleaca C., Albu L., Savoiu M., Sandu I., Popovici E., and Soare I., 2005. Iron-iron oxide core-shell nanoparticles synthesized by laser pyrolysis followed by superficial oxidation. Applied Surface Science, Vol. 247, pp. 25-31.

Gangopadhayay P., Gallet, S., Franz, E., Persoons, A., and Verbiest, T., 2005. Novel superparamagnetic Core (Shell) nanoparticles for magnetic targeted drug delivery and hyperthermia treatment. IEEE Transactions of Magnetics, Vol. 41, pp. 4194-4196.

Gangopadhayay S., Hadjipanayis G.C., Shah S.I., Sorensen C.M., Klabunde K.J., Papaefthymiou V., and Kostikas A., 1991. Effect of oxide layer on the hysteresis behavior of fine Fe particles. Journal of Applied Physics, Vol. 70, pp. 5888-5890.

Hilger I., Hilger I., Kießling A., Romanus E., Hiergeist R., Hergt R., Andrä W., Roskos M., Linss W., Weber P., Weitschies W., and Kaiser W. A., 2004. Magnetic nanoparticles for selective heating of magnetically labelled cells in culture: preliminary investigation. Nanotechnology, Vol. 15, pp. 1027-1032.

Kamat P.V., 2002. Photophysical, photochemical and photocatalytic aspects of metal nanoparticles. Journal of Physical Chemistry B, Vol. 106, pp. 7729-7744.

Klein M.W., Tritschler T., Wegener M. and Linden S., 2005. Lineshape of harmonic generation by metallic nanoparticles and metallic photonic crystal slabs. Physical Review B, Vol. 72, pp. 115113-115124.

Kozicki M.N., Mitkova M., Park M., Balakrishnan M., and Gopalan C., 2003. Information storage using nanoscale electrodeposition of metal in solid electrolytes. Superlattice and Microstructure, Vol. 34, pp. 459-465.

Meiklejohn W.H. and Bean C.P., 1957. New magnetic anisotropy. Physical Review, Vol. 105, pp. 904-913.

Nowack B. and Bucheli T., 2007. Occurrence, behavior and effects of nanoparticles in the environment. Environmental Pollution, Vol. 150, pp. 5-22.

Presting H., and Konig U., 2003. Future nanotechnology developments for automotive applications. Material Science Engineering: C, Vol. 23, pp. 737-741.

Roumenin C.S., 1994. Solid State Magnetic Sensors (Elsevier, Amsterdam)

Roy M.K., Nambissan P.M.G., and Verma H.C., 2002. Structural, thermal stability and defect studies of Fe-Ag alloy prepared by electrodeposition technique. Journal of Alloys and Compounds, Vol. 345, pp. 183-188.

Roy M.K., Subrahmanyam V.S., and Verma H.C., 2004. Defect studies in Fe-Cu alloys prepared by electrodeposition. Physics Letters A, Vol. 328, pp. 375-379.

Shah V.V., Basu A., 2008. Thin Film Technology - A Layman’s Perception (Vigyan Prasar, India).

Tuček J., Zboril R., and Petridis D., 2006. Maghemite nanoparticles by view of Mossbauer spectroscopy. Journal of Nanoscience and Nanotechnology, Vol. 6, pp. 926-947.

Biographical notes

Dr. Santosh Kumar is a faculty member in the Physics Department of B S College, Danapur in Bihar, primarily meant to provide undergraduate education. He has established a research laboratory in his college where problems related to nanoparticle synthesis are investigated.

Samar Layek had completed his B.Sc. in 2005 from Calcutta University and M. Sc. in 2007 from Indian Institute of Technology, Kanpur, India. At present he is perusing doctoral degree from Indian Institute of Technology, Kanpur, India under joint supervision of Prof. H. C. Verma and Dr. Zakir Hossain. 
Dr. Brajesh Pandey completed his doctoral degree from Indian Institute of Technology, Kanpur, India in 2008. His area of research includes defect production in metallic alloy system using mechanical deformation, nanoparticle oxides and geological boundaries.

Prof. H. C. Verma completed his doctoral degree from Indian Institute of Technology, Kanpur in 1981. He has 85 research papers to his credit and has supervised eight PhD students. Currently he is Professor of Physics at IIT Kanpur working in the areas of nanomaterials and nanofabrication.

Received June 2010

Accepted November 2010

Final acceptance in revised form November 2010 